\title{
Green synthesis, characterization and biological activities of silver nanoparticles using the bark extract of ailanthus altissima
}

\author{
Maria Arshad ${ }^{1}$, Abbas Khan ${ }^{2, *}$, Zahoor H. Faroodi ${ }^{4}$, Muhammad Usman $^{3}$, \\ M. AbDul WASEem ${ }^{1}$, SAYyar Ali ShaH ${ }^{2}$, MOMIN KhaN ${ }^{2}$ \\ ${ }^{1}$ Department of Chemistry, Sarhad University of Science and Information Technology Peshawar, Pakistan \\ ${ }^{2}$ Department of Chemistry, Abdul Wali Khan University Mardan, 23200 Pakistan \\ ${ }^{3}$ Department of Chemistry, Government College University, Faisalabad, Pakistan \\ ${ }^{4}$ Institute of Chemistry, University of the Punjab, New Campus, Lahore, Pakistan
}

\begin{abstract}
Due to their potential application in various fields of science and technology, the eco-friendly bio-synthesis of silver (Ag) nanoparticles (NPs) is a growing area for researchers. In this study, we report the green synthesis of Ag nanoparticles and their characterization by using various techniques. For the preparation of $\mathrm{Ag}$ particles, aqueous plant extract of ailanthus altissima was used as a reducing medium for $\mathrm{Ag}^{+}$ions of silver nitrate to $\mathrm{Ag}^{0}$. UV-Vis spectrophotometry was used to trace the formation of $\mathrm{Ag}$ particles by noting their surface plasmon resonance peaks ( $400 \mathrm{~nm}$ to $440 \mathrm{~nm}$ ). Fourier transform infrared spectroscopy (FT-IR) was employed to reveal the chemical composition of Ag nanoparticles which were capped by plant extract. Scanning electron microscopy (SEM) was used to get the lattice image, morphology and average size of Ag particles. The average size distribution of Ag NPs dispersed in aqueous media was also measured using dynamic light scattering (DLS). It was found that DLS results are in good agreement with those obtained from SEM. The synthesized particles were then subjected to the antibacterial and antifungal activities by studying them against various species, such as bacillus cereus, staphylococcus aureus, pseudomonas aeruginosa, E. coli and A. parasiticus, A. niger and A. flavus fungi. It was noted from the growth curves of both bacteria and fungi that in the presence of silver nanoparticles they show more in-zone growth as compared to the plant extract.
\end{abstract}

Keywords: ailanthus altissima; silver nanoparticles; green synthesis; SEM; biological activitie

\section{Introduction}

The science and technology related to fine particles belong to the advanced and respected areas of research. Nanotechnology is dealing with matter at the atomic or molecular level. The scientists all over the world are now considering forthcoming effects of nanotechnology on human and environment. Nanotechnology is likely to be crucial to the $21^{\text {st }}$ century scientific and medical advancements and its developments will be determined by the collective hard work of natural, physical, medical, engineering and related sciences [1]. The materials in nanoscale can show different properties as compared to what they exhibit on a macroscale.

*E-mail: abbas053@gmail.com
For example, gold is chemically inactive at standard conditions, but it acts as a strong chemical promoter at nanoscale. The recent developments in the nanotechnology are attributed to the surface modifications shown by matter at nanoscale. A significant feature of nanotechnology is the improvement of new procedures for the production of nanoparticles of altered chemical compositions, shapes, sizes and well-ordered disparity [2].

The recent development in the field of nanotechnology brought the identification of unexpected beneficial properties of metal nanoparticles such as gold, platinum, palladium and silver nanoparticles. Au NPs are quite stable, inert and their electronic, magnetic and optical properties can be adjusted. Au NPs have been utilized by coupling to biomolecules for medical 
applications. For example, they can detect DNA with high sensitivity and selectivity and have potential applications as contrast agents in cancer diagnosis and therapy as well as mutations, single nucleotide polymorphisms (SNPs), chromosomal translocations, gene expression and pathogens from clinical samples [3].

Due to the large surface area that hinders microbial growth compared to silver metal in a bulk form, the silver nanoparticles have strong and wide spectrum of antimicrobial activities [4]. Another important property of $\mathrm{Ag}$ particles is that they are nontoxic to microorganisms at low concentration and have negligible side effects [5]. Silver nanoparticles have many applications in ecology and medicine, purification and filtering of water technologies and in the preparation of various creams to prevent infection of burns and open wounds [6]. Because of various applications, the preparation, characterization and physicochemical studies of Ag nanoparticles is of prime importance. There are three common methods: physical, chemical, and biological, which are generally used for the synthesis of metal nanoparticles. The most common chemical method is important for the production of pure and well defined nanoparticles but it is not cost effective neither eco-friendly. This is because it requires severe conditions such as high temperature, high pressure and toxic chemicals such as organic solvents and toxic reducing agents. Unlike the chemical method, the green synthetic concept is more advantageous because it does not require elaborate processes, such as intracellular synthesis and numerous purification steps, high pressure, energy and toxic chemicals, and, therefore, it is cost effective and environmentally friendly [7]. Several plants have been utilized for the production of silver nanoparticles. In this paper, we have reported the synthesis and characterization of silver nanoparticles using an aqueous extract of ailanthus altissima. Due to the presence of various important medical compounds in this plant, it is supposed that $\mathrm{Ag}$ nanoparticles capped ailanthus altissima may be useful from academic as well as applied side $[8,9]$. Furthermore, to the best of our knowledge, this may be the first report on the preparation and capping of Ag nanoparticles using the aqueous extract of ailanthus altissima.

\section{Experimental}

\subsection{Reagents and equipment}

Silver nitrate was purchased from Sigma Aldrich and double distilled water was used for all reactions, solutions preparation, and analysis purposes. FT-IR spectra were recorded on IR Affinity (WQF 510\520 model). UV-Vis spectrophotometry was carried out with Shimadzu 1800 model. Particle morphology and average size were investigated using SEM (FEI Quanta FEG 200) under a high and low vacuum while the size distribution of $\mathrm{Ag}$ particles was determined using particle size analyzer (Malvern Instruments). The antibacterial and antifungal activities were studied against bacillus cereus, pseudomonas aeruginosa, E. coli and $A$. parasiticus, A. niger and A. flavus fungi.

\subsection{Collection of plant material and preparation of its extract}

The barks of ailanthus altissima were collected from the trees grown in the botanical garden of Pakistan Forest Institute Peshawar, Pakistan. The samples were brought to the laboratory in polythene bags and were cleaned thoroughly with distilled water to remove the dust and other microbes. This plant is also called as tree-of-heaven and is one of the highly medicinal plants considered to be one of the precious assets in the medicinal plant market. Due to its importance in the herbal medicine market it is highly desired to be used for green synthesis. After collection of ailanthus altissima plant, its barks were cleaned thoroughly with distilled water and shade dried. Then the barks were crushed into small pieces and $10 \mathrm{~g}$ of them was taken in a conical flask and soaked in $100 \mathrm{~mL}$ of distilled water. The soaked barks were boiled for $15 \mathrm{~min}$ to $20 \mathrm{~min}$, allowed to cool and then filtered through Whatman filter paper to get a clear extract. 


\subsection{Preparation of silver nanoparticles}

For the preparation of Ag nanoparticles, aqueous solutions of silver nitrate and plant extract with different ratios were used. For this purpose, the earlier procedure for the synthesis of metal nanoparticles was adopted [10]. In a typical experiment, about $0.02 \mathrm{~g}$ of silver nitrate $\left(\mathrm{AgNO}_{3}\right)$ was dissolved with a specific amount of the plant extract in a $100 \mathrm{~mL}$ volumetric flask and diluted up to the mark with distilled water. The reaction mixture was kept on stirring for 4 to 5 hours at room temperature. The change in color, which gives a primary indication of nanoparticles formation, was observed visually for each reaction. The color intensity of the samples was found to change with changing various experimental parameters. Silver nanoparticles were isolated by centrifugation at $14000 \mathrm{rpm}$ and the solid Ag NPs were collected for further characterization. The synthesized nanoparticles were then characterized using UV-Vis spectrophotometry, Fourier transform infrared spectroscopy (FT-IR), particle size analyzer (Malvern instruments), and scanning electron microscopy (SEM).

\section{Results and discussion}

\subsection{Characterization with UV-Vis spec- troscopy}

The successful preparation of Ag NPs was confirmed by noting the color changes through visual observation. In addition to this, the UV-Vis spectrophotometry was used to trace the formation of $\mathrm{Ag}$ particles by noting their surface plasmon resonance peaks (400 nm to $440 \mathrm{~nm}$ ). The color of reaction mixture has changed not only upon synthesis of Ag nanoparticles with the bark extract of ailanthus but it was also affected by varying the ratio of salt to plant $\left(\mathrm{AgNO}_{3}\right.$ :ailanthus). Addition of bark extract with $\mathrm{AgNO}_{3}$ in a specific ratio changed the color of the mixture solution from white to yellow and finally to dark orange. This change in color can be attributed to the formation of Ag NPs of different shapes and sizes as the ratio of the reaction mixture is changing. In other words, this may be due to the property of quantum confinement and size dependent property of nanoparticles affecting the physical properties of the nanoparticles. Intensity of color was found to be the highest at 10:1 ratio, therefore, this ratio has been considered as an optimum ratio for synthesis of Ag NPs.

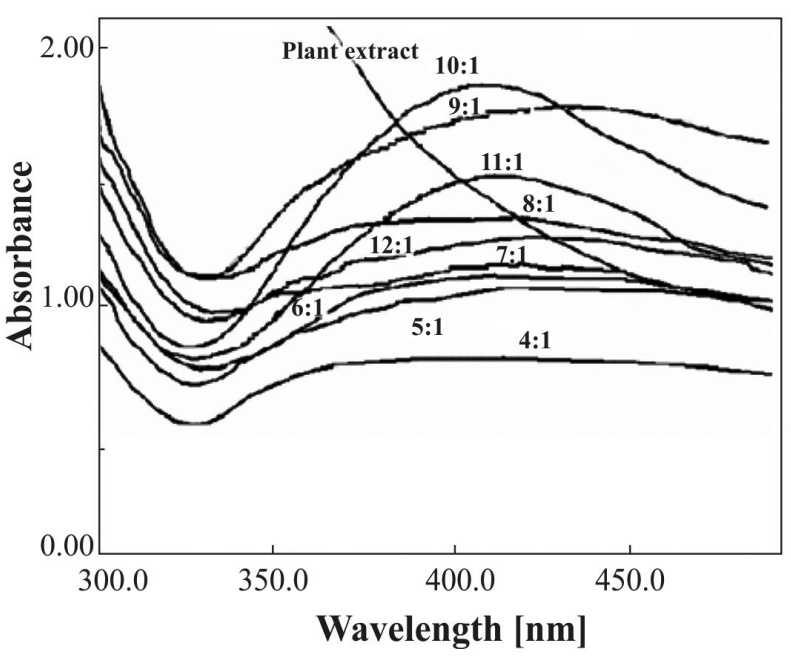

Fig. 1. UV-Vis spectra of ailanthus altissima bark extract alone and with $\mathrm{AgNO}_{3}$ solution mixed in different ratios.

Fig. 1 shows the UV-Vis absorption spectra of the samples prepared by mixing different amounts of $\mathrm{AgNO}_{3}$ solution with the fixed amount of ailanthus bark extracts. It can be seen that the peak position, intensity of the peaks and shape of the spectrum is changing by changing the ratio. Moreover, the absorption spectrum of ailanthus bark extracts alone was also examined but did not give any peak in the visible region (the figure is not shown here). Fig. 1 also shows that by mixing the silver nitrate solution with the plant extract gives the peak near $415 \mathrm{~nm}$ which confirms the formation of silver nanoparticles and is consistent with the reported literature [6]. This figure further reveals that the intensity of the absorbance band of the mixture of silver nitrate solution with aqueous plant extract in 10:1 ratio is the highest and can be considered as an optimum ratio. Therefore, a mixture of bark extract and $\mathrm{AgNO}_{3}$ solution in 10:1 ratio was prepared in bulk and used for further study of silver nanoparticles. 


\subsection{Fourier transform infrared spec- troscopy of silver nanoparticles}

The vibrational spectroscopy was used to trace various functional groups corresponding to different phytochemicals present in the bark extract of ailanthus altissima alone and to see the changes in the plant composition after synthesis of Ag NPs in this extract. FT-IR spectra of the plant alone and silver NPs-capped with the plant extract are shown in Fig. 2a and Fig. 2b, respectively. Various IR peaks related to different functional groups have been obtained at various peak positions. FT-IR was used to obtain the information about chemical composition of the synthesized Ag NPs. FT-IR gives the information about functional groups present in the synthesized silver nanoparticles to understand their transformation from simple inorganic $\mathrm{AgNO}_{3}$ to elemental silver by the action of different phytochemicals present in the plant. These phytochemicals are supposed to act simultaneously as reducing, stabilizing and capping agents for the nanoparticles. A general comparison of these figures reflects also the successful bio-fabrication of silver nanoparticles mediated by the plant extracts as the shapes and positions of the IR peaks are somehow changed for $\mathrm{Ag}$ NPs-capped with the extract compared to the extract alone. An analysis of these peaks shows that the peaks at $2962.0 \mathrm{~cm}^{-1}, 1414.5 \mathrm{~cm}^{-1}$, $1258.0 \mathrm{~cm}^{-1}, \quad 1010.0 \mathrm{~cm}^{-1}, \quad 863.9 \mathrm{~cm}^{-1}$, $787.9 \mathrm{~cm}^{-1}$ and $686.5 \mathrm{~cm}^{-1}$ are due to amide linkage. The peak at $1539 \mathrm{~cm}^{-1}$ is due to carbonyl stretching of the nanoparticle. The peaks at $977.7 \mathrm{~cm}^{-1}, 966.0 \mathrm{~cm}^{-1}$ and $798.4 \mathrm{~cm}^{-1}$ correspond to carbon nitrogen stretching vibrations in amine. These phytochemicals are supposed to play simultaneously a role of reducing, stabilizing and capping agents for the nanoparticles.

\subsection{SEM and DLS analysis of silver nanoparticles}

The surface morphology and approximate size of silver nanoparticles was determined by scanning electron microscopy (SEM). Fig. 3a and Fig. 3b show SEM micrographs of Ag NPs capped with ailanthus at different resolutions. These figures

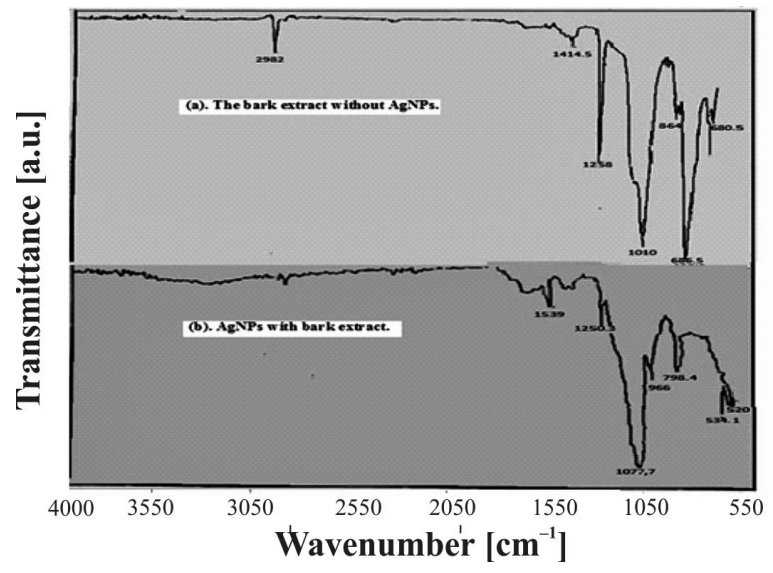

Fig. 2. (a) FT-IR spectra of the bark extract without Ag NPs. (b) FT-IR spectra of Ag NPs with bark extract.

reflect that relatively spherical and uniform Ag NPs with diameters ranging from $46 \mathrm{~nm}$ to $80 \mathrm{~nm}$ were formed. Some of the larger silver particles may have been formed due to the aggregation of the smaller molecules. It is assumed that the capping of silver NPs with bioorganic molecules may be due to interactions of hydrogen bonds between the phytochemicals and Ag NPs.

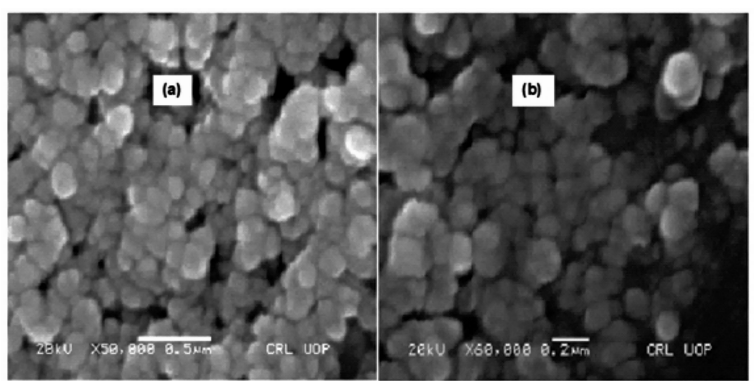

Fig. 3. SEM micrographs of Ag NPs capped with the bark extract of ailanthus altissima at different magnifications; white bar $=0.5 \mu \mathrm{m}$ (a) and $0.2 \mu \mathrm{m}$ (b).

Likewise, to determine the average size of $\mathrm{Ag}$ NPs, dynamic laser light scattering (DLS) measurements were carried out on a standard Malvern Zetasizer, NANO ZS (Malvern Instruments Limited, UK), using a $\mathrm{He}-\mathrm{Ne}$ laser with a wavelength of $633 \mathrm{~nm}$ and a detector angle of $90^{\circ}$. The dispersion of Ag NPs was passed through Millipore Millex-HV filters with a pore size of $0.80 \mu \mathrm{m}$ to 
remove dust before the DLS measurements. A typical intensity fraction distribution versus diameter of the Ag NPs dispersion is shown in Fig. 4. It is seen that the value of scattered intensity is varying with population of Ag NPs with the corresponding size. The average size of the particles was assessed from the maxima of intensity-diameter profile. It can be seen that this average size of Ag NPs dispersion is approximately equal to $80 \mathrm{~nm}$. It can be said that the DLS results are in good agreement with those obtained from SEM.

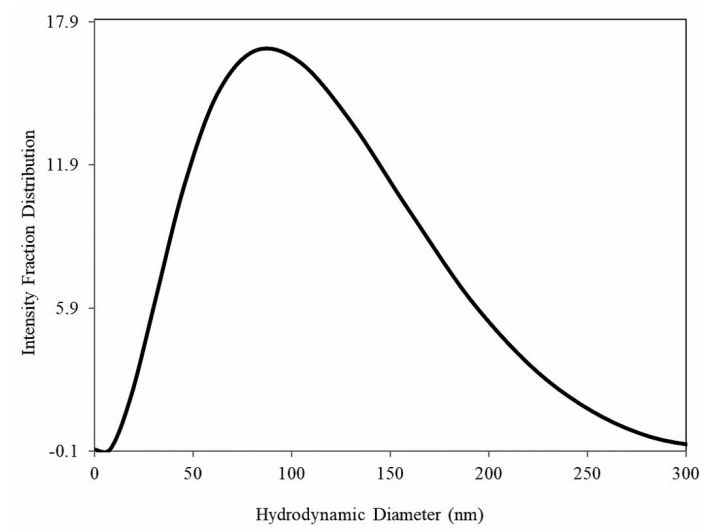

Fig. 4. Representative plot of intensity fraction distribution versus apparent hydrodynamic diameter for aqueous solutions of Ag NPs stabilized with the bark extract of ailanthus altissima at room temperature.

\subsection{Biological activities of Ag NPs disper- sion}

It is now known that silver NPs show good anticancer and antioxidant activities [12], therefore, the antibacterial and antifungal activities of the bark extract of ailanthus altissima alone and Ag NPs capped and stabilized by the extract of ailanthus altissima have also been investigated and their comparative results are discussed.

Antibacterial activity: The antibacterial activity of $\mathrm{AgNP}_{\mathrm{s}}$ and bark extract of the plant were determined and the results are shown in Table 1. A well diffusion method was used for determining the antibacterial activity. Comparing the activities of the plant without NPs and NPs capped with the plant extract presented in Table 1, it can be seen that the pure bark extract is less effective than the nanoparticles synthesized using the plant extract. Furthermore, the results reveal that the nanoparticles show high inhibition zone against different types of bacteria (staphylococcus aureus, pseudomonas aeruginosa, E. coli) as compared to the bark extract which shows less inhibition zone. In addition to this, Table 1 also reveals that in case of staphylococcus aureus bacteria, the nanoparticle shows $22 \mathrm{~mm}$ zone of inhibition while in aqueous bark extract $15 \mathrm{~mm}$ zone of inhibition was determined. Similarly, the nanoparticle shows $27 \mathrm{~mm}$ zone of inhibition against pseudomonas aeruginosa, whereas the extract shows $20 \mathrm{~mm}$ zone of inhibition. Likewise, the Ag NPs show $25 \mathrm{~mm}$ zone of inhibition against $E$. coli bacteria while the bark extract shows $17 \mathrm{~mm}$ zone of inhibition. It is concluded that the synthesized Ag NPs have high antibacterial activity against different strains as compared to the plant alone.

Antifungal activities: Table 2 shows the response of bark extract and silver nanoparticles synthesized by green methods using the same plant extract, against different types of fungi, (A. parasiticus, A. niger and A. flavus). The results reveal that the silver nanopracticles show larger zone of inhibition against fungi as compared to the bark extract. When using A. parasiticus fungi, the silver nanoparticles show $20 \mathrm{~mm}$ zone of inhibition, while the bark extract shows $13 \mathrm{~mm}$ zone of inhibition. This table further shows that the bark extract shows $15 \mathrm{~mm}$ zone of inhibition against $A$. nigar fungi as compared to the nanoparticle which shows $9 \mathrm{~mm}$ zone of inhibition. The silver nanoparticles show $9 \mathrm{~mm}$ zone of inhibition against $A$. flavus fungus but the bark extract shows $5 \mathrm{~mm}$ zone of inhibition. Thus, it can be concluded that Ag nanoparticles have better antifungal activities as compared to the bark extract of ailanthus altissima.

\section{Conclusions}

On the basis of the performed investigation, it is concluded that ailanthus altissima plant extract shows exceptional reducing and stabilizing abilities for Ag NPs synthesis. This property of the plant 
Table 1. Antibacterial activity of the bio-synthesized silver nanoparticles and the bark extract of ailanthus altissima alone.

\begin{tabular}{lcc}
\hline Name of the bacteria & $\begin{array}{c}\text { Zone of inhibition of } \\
\text { synthesized nanoparticle [mm] }\end{array}$ & $\begin{array}{c}\text { Zone of inhibition of aqueous } \\
\text { plant extract [mm] }\end{array}$ \\
\hline \hline Staphylococcus aureus & 22 & 15 \\
Pseudomonas aeruginosa & 27 & 20 \\
E. coli & 25 & 17 \\
\hline
\end{tabular}

Table 2. Antifungal activity of the bio-synthesized silver nanoparticles and the bark extract of ailanthus altissima alone.

\begin{tabular}{lcc}
\hline Name of fungus & $\begin{array}{c}\text { Zone of inhibition of } \\
\text { synthesized nanoparticles } \\
{[\mathrm{mm}]}\end{array}$ & $\begin{array}{c}\text { Zone of inhibition of bark } \\
\text { extract [mm] }\end{array}$ \\
\hline \hline A. parasiticus & 20 & 13 \\
A. nigar & 15 & 9 \\
A. flavus & 9 & 5 \\
\hline
\end{tabular}

extract is assigned to the presence of phytochemical compounds, such as aldehyde and amine type moieties in the plant extract, which help in the reduction of $\mathrm{Ag}^{+3}$ to $\mathrm{Ag}^{0}$. This conclusion can be drawn from the FT-IR results which show the presence of different types of groups present in the plant extract, such as amide linkage, carbonyl linkage and hydroxyl linkage. The UV-Vis spectra (in $300 \mathrm{~nm}$ to $600 \mathrm{~nm}$ wavelength region) also confirm the successful fabrication of Ag NPs using this plant extract. Furthermore, from UV-Vis results, it was found that the optimum ratio for the most effective and stable synthesis of AgNPs was 10:1 (plant/salt). The SEM and DLS analyses revealed that the average size of the synthesized AgNPs was approximately $80 \mathrm{~nm}$. SEM results also showed that the particles had a good and acceptable morphology. The antibacterial and antifungal activities of the bark extract of ailanthus altissima alone and Ag NPs capped and stabilized with the extract of ailanthus altissima were also investigated and the comparison of the results showed that the nanoparticles were more effective against different types of bacteria and fungi as compared to the bark extract itself.

\section{References}

[1] Lubick B.N., Environ. Sci. Technol., 11 (2008), 3910.
[2] Shankar S.S., Ahmed A., Pasricha R., SasTRY M., J. Mater. Chem., 7 (2003), 1822.

[3] Hewakuruppu Y.L., Dombrovsky L.A., Chen C., Timchenko V., Jiang X., Appl. Optics., 24 (2013), 6041.

[4] Zhang Y., Yang D., Kong Y., Wang X., GaO G., Nano. Biomed. Eng., 4 (2010), 252.

[5] Malabadi R.B., Meti N.T., Mulgund G., Nataraja K., Kumar S.V., Res. Plant Biol,, 5 (2012), 32.

[6] Song J.Y., KIM B.S., Bioprocess Biosyst. Eng., 1 (2009), 79.

[7] Chandran S.P., Chaudhary M., Pasricha R., Ahmad A., Sastry M., Biotechnol. Progr., 2 (2006), 577.

[8] Feo V.D., Martino L.D., Quaranta E., Pizza C., J. Agric. Food Chem., 5 (2003), 1177.

[9] Bray D.H., Boardman P., O’Neill M.J., Chan K.L., Phillipson J.D., Warhurst D.C., SuffNESS M., Phytother. Res., 1 (1987), 22.

[10] Kimling J., Maier M., OKenve B., Kotaidis V., Ballot H., Plech A., J. Phys. Chem. B., 32 (2006), 15700.

[11] Raja P.B., Rahim A.A., Qureshi A.K., Awang K., Mater Sci.-Poland, 3 (2014), 408.

[12] Kumar B., Smita K., Cumbal L., Debut A., Camacho J., Hernández-Gallegos E., Chávez-López M.G., Grijalva M., Angulo Y., Rosero G., Adv. Mat. Lett., 2 (2015), 127. 